Provided for non-commercial research and education use. Not for reproduction, distribution or commercial use.

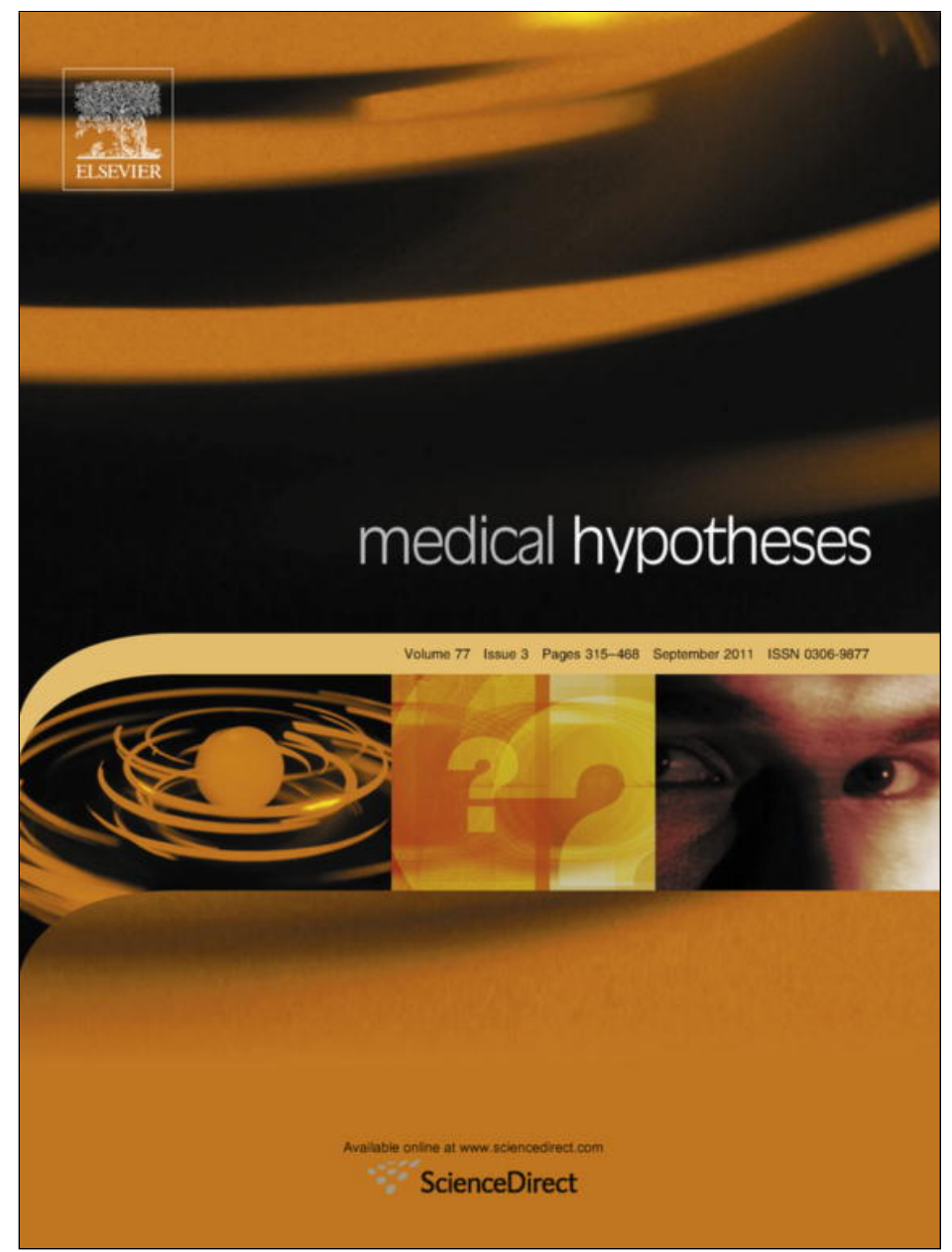

This article appeared in a journal published by Elsevier. The attached copy is furnished to the author for internal non-commercial research and education use, including for instruction at the authors institution and sharing with colleagues.

Other uses, including reproduction and distribution, or selling or licensing copies, or posting to personal, institutional or third party websites are prohibited.

In most cases authors are permitted to post their version of the article (e.g. in Word or Tex form) to their personal website or institutional repository. Authors requiring further information regarding Elsevier's archiving and manuscript policies are encouraged to visit:

http://www.elsevier.com/copyright 


\title{
Information homeostasis as a fundamental principle governing the cell division and death
}

\author{
Arvind Kumar ${ }^{\mathrm{a}, *}$, Harinder Pal Singh ${ }^{\mathrm{b}}$ \\ ${ }^{a}$ Bernstein Centre Freiburg, and Neurobiology and Biophysics, Faculty of Biology, University of Freiburg, Freiburg, Germany \\ ${ }^{\mathrm{b}}$ Dept. of Medical Oncology, Command Hospital, Lucknow, India
}

\section{A R T I C L E I N F O}

\section{Article history:}

Received 1 November 2010

Accepted 2 May 2011

\begin{abstract}
A B S T R A C T
To express the genetic information with minimal error is one of the key functions of a cell. Here we propose an information theory based, phenomenological model for the expression of genetic information. Based on the model we propose the concept of 'information homeostasis' which ensures that genetic information is expressed with minimal error. We suggest that together with energy homeostasis, information homeostasis is a fundamental working principle of a biological cell. This model proposes a novel explanation of why a cell divides and why it stops to divide and, thus, provides novel insights into oncogenesis and various neuro-degenerative diseases. Moreover, the model suggests a theoretical framework to understand cell division and death, beyond specific biochemical pathways.
\end{abstract}

(c) 2011 Elsevier Ltd. All rights reserved.

\section{Introduction}

Why does a cell divide? The textbook explanation is that a cell divides to keep its volume to surface area ratio constant. However, this simple metabolism based argument for cell division fails to explain why, for instance, cancer cells continue to divide. Multiple biochemical pathways and signalling cues have been identified that initiate cell division [1] and apoptosis [2]. However, these mechanisms only explain 'how' a cell decides to divide or not, and fail to answer the question as to 'why' a cell must divide, particularly when it is part of a larger cell population (e.g. a tissue). Although, the idea of maintaining the surface to volume ratio does not explain 'how' a cell divides but it is very powerful in providing a reason for cell division. The idea of maintaining volume to surface area ratio constant, is a single cell based mechanism and it is possible that when a cell is a part of a multi-cellular environment, new general and simple rules emerge to govern cell division and apoptosis. A conceptual understanding of the process of cell division is necessary not only to unify the diverse biochemical and signalling mechanisms governing cell division and apoptosis but also to understand the mechanisms underlying various cancers and degenerative diseases.

Here, we consider a cell (isolated or part of a multi-cellular environment) as a system that strives to express the genetic information it carries in its nucleus, depending on its lineage and environmental cues. Clearly, a cell performs many tasks when part of a tissue, still, most of the tasks performed by a cell require expression of specific genes. Specifically, we draw an analogy between

\footnotetext{
* Corresponding author.

E-mail address: arvind.kumar@biologie.uni-freiburg.de (A. Kumar).
}

a typical electrical communication system and a biological tissue. Using a phenomenological model of genetic information expression we introduce the concept of 'information homeostasis' for a cell population (e.g. tissue or organ) as one of the fundamental principles governing cell functioning in a multi-cellular environment. We argue that cell division or death is a way to maintain 'information homeostasis' in a tissue/organ and hypothesize that disturbances in the 'information homeostasis' underlie uncontrolled cell division or apoptosis in a tissue.

\section{Information theory based model of flow of genetic information}

Before describing our model of expression of genetic information we give a brief introduction of a digital communication system. This is necessary to introduce few terms and a basic result of the communication theory.

\section{A brief introduction to digital communication system}

In a digital communication system, there are three main components: a source, which has something to transmit e.g. text data, a channel through which the information from the source is transmitted e.g. a set of optical fibres, and a receiver e.g. a computer with appropriate interface to the optical fibres (fig. 1A). The source has a certain capacity to generate/transmit information (source capacity $\boldsymbol{S})$. Similarly, the data transmission properties of the channel are quantified as the (channel capacity $\boldsymbol{C}$ ). Typically, the source and channel capacity are expressed as bits/s. The second theorem of Shannon puts an upper bound on the source capacity, given a channel, to ensure an error free transmission [3]. According to this theorem for a given channel, when the source capacity is less than or 
A

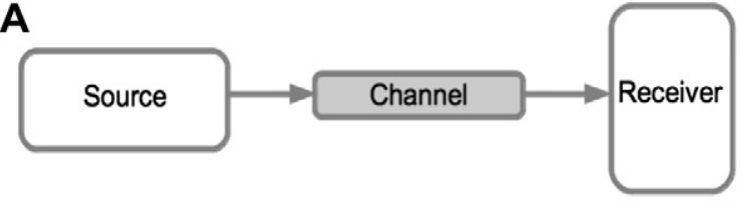

B

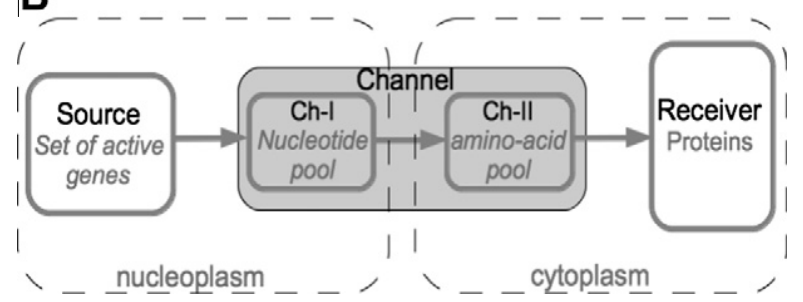

Fig. 1. Phenomenological model of flow of genetic information. (A) Schematic of a digital communication system. (B) An schematic of an information theory based model of flow of genetic information from DNA to proteins. The DNA in the nucleus of the cell is the source of information. The source capacity is determined by the set of active genes. The genetic information is transmitted over a two stage channel. The first stage involves the nucleotide pool (Ch-I) and through the process of transcription mRNAs are formed. This intermediated stage of the information is sent to the cytoplasm and uses the amino-acid pool. The process of translation is carried out in the second stage (Ch-II) of the channel and results in proteins.

equal to the channel capacity (i.e. $\boldsymbol{S} \leqslant \boldsymbol{C}$ ) [theorem 11 in Ref. [3]] the information flows without error. Thus, to ensure an error-free transmission, if the source capacity is increased for some reason, then it is important to increase the channel capacity accordingly. The channel capacity can be increased by changing the channel itself, for example optical fibres have greater channel capacity than the copper wires. Additionally, by laying down more channels and dividing the source information over each channel according to their individual channel capacities, overall capacity of the channel can be increased. Both solutions are popular in the communications industry. However, once a particular channel is chosen, it is simpler to increase the number of channels in order to increase the overall channel capacity.

\section{Cell as an information transmission system}

In a multi-cellular organism, although each cell harbours a complete copy of the organism's genome, it expresses only a small subset of the genomic information into proteins depending on the cell type, its lineage and environmental cues [4]. In the following we draw an analogy between the flow of genetic information from DNA to proteins and the information transmission in a digital communications system (fig. 1B) [3].

\section{Source and source capacity}

The DNA in the nucleus of the cell is the source of information in the cell. Functional specialization of cells in a multi-cellular organism implies that only a small subset of the whole genome is active in each cell. In fact, cell specific expression of genes starts very early in the embryo [4]. The set of active genes in the cell determines the source capacity $\boldsymbol{S}$ of a cell in a multi-cellular environment. That is, $\boldsymbol{S}$ depends on the distribution of nucleotide bases and amino-acids that are necessary to express the active genes. $\boldsymbol{S}$ is a dynamic entity and environmental cue dependent activation/ inactivation of genes, change in proteosomal activity, and developmental stage of a cell can alter $\boldsymbol{S}$. In multi-cellular organisms individual source capacities sum up to the source capacity of the whole genome.
Channel and channel capacity

The expression of genetic information requires two well described steps i.e. 'transcription' and 'translation', which take place in nucleoplasm and cytoplasm, respectively. Cytoplasm also acts as the receiver of the genetic information in the form of proteins (fig. 1B).

Thus, nucleoplasm and cytoplasm make a two stage channel for the transmission of genetic information. The nucleotide pool $(\mathbf{N}$ pool) in the nucleus acts as a channel for the process of 'transcription' and, the amino-acid pool (AA-pool) constitutes the channel for the process of 'translation'. Thus, genetic information flows from genes to mRNA through the pool of nucleotides ( $\mathbf{N}$-pool, Ch-I) and from mRNA to proteins through the pool of amino-acids (AA-pool, Ch-II).

The channel capacity $\boldsymbol{C}_{\mathbf{1}}$ of the $\boldsymbol{N}$-pool is dependent on the composition of nucleotides in the $\mathbf{N}$-pool. Similarly, the channel capacity $\boldsymbol{C}_{\mathbf{2}}$ of the $\boldsymbol{A} \boldsymbol{A}$-pool is dependent on the composition of amino-acids in the $\boldsymbol{A} \boldsymbol{A}$-pool (see below for details). Because the genetic information flows in a serial fashion over the $\boldsymbol{C h}-\mathbf{1}$ and $\boldsymbol{C h}$-II, the effective channel capacity $\boldsymbol{C}_{\text {eff }}$ is equal to $\min \left(\boldsymbol{C}_{\mathbf{1}}, \boldsymbol{C}_{\mathbf{2}}\right)$.

\section{Information homeostasis: conditions for an error-free expression of genetic information}

In a noisy information transmission channel to ensure an error free transmission of information, channel capacity should be greater than or equal to the source capacity i.e. $\boldsymbol{S} \leqslant \boldsymbol{C}_{\text {eff }}$ (3). Because, genetic information transmission is largely error free [5-7], we can assume that in the cell Shannon's second theorem holds true [3]. Thus, in a healthy multi-cellular organism every cell is in an 'information homeostasis' and $\boldsymbol{S}$ and $\boldsymbol{C}_{\text {eff }}$ are adjusted to ensure transmission/expression of the genetic information with minimum error [5-7].

Because cell division creates additional $\boldsymbol{A} \boldsymbol{A}$-pool and $\mathbf{N}$-pool we hypothesize that it adds new channels $\left(\boldsymbol{C}_{\mathbf{1}}\right.$ and $\boldsymbol{C}_{\mathbf{2}}$ ). However, such new channels would increase $\boldsymbol{C}_{\text {eff }}$ only when the information load of a cell population can be redistributed over new channels. Here, the asymmetric cell division is particularly important because such division could be used as a mechanism to redistribute the information load differentially between the daughter cells [8-10]. Thus, the asymmetric cell division can be regarded as a mechanism to increase the $\boldsymbol{C}_{\text {eff. }}$ Similarly, cell death, because it removes existing channels, would result in a reduction in the $\boldsymbol{C}_{\text {eff. }}$. We suggest that continuous cell division and death is a mechanism by which 'information homeostasis' is maintained in a cell population. Thus, according to this model, besides the energy homeostasis, a healthy cell, as a part of a cell population, also maintains its information homeostasis.

$N$-pool and AA-pool composition as an indicative of the channel capacity

In the 'transcription' process, in a statistical sense, the task the cellular machinery has to solve is to find a complementary nucleotide base corresponding to the one in the gene being transcribed, in a limited time. Thus, the ease with which a complementary nucleotide can be found is related to the channel capacity of the $\boldsymbol{N}$-pool. In statistical terms, when the distribution of the nucleotide bases in the nucleus (composition of $\mathbf{N}$-pool) is matched with the distribution of nucleotide bases in the active genes, it would be the easiest to find a complementary nucleotide base. Cellular machinery solves a related problem in the cytoplasm during the process of 'translation' and likewise, the ease with which a tRNA can find a matching amino-acid is associated with the channel capacity of the $\boldsymbol{A} \boldsymbol{A}$-pool. Thus, distribution of free nucleotides in 
the nucleus and amino-acids in the cytoplasm is an indicative of the channel capacity of a cell.

Because a cell is able to replicate its genome during cell division, we assume that the distribution of the nucleotide bases in the nucleus is optimal for the genome. However, note that, cell division and replication of the genome is not the same as the flow of genetic information from genes to proteins as it is possible that nucleotide distribution optimal for genome replication is sub-optimal for a given set of active genes. This is not the case with the $\boldsymbol{A A - p o o l}$ and thus, we argue that the composition of the $\boldsymbol{A} \boldsymbol{A}$-pool (distribution of the amino-acids in the cytoplasm) is more critical for the flow of genetic information.

In summary, the information homeostasis is a state in which the distribution of nucleotides in the nucleus and amino-acids in the cytoplasm is closely matched with the nucleotide and amino-acid requirements of the active genes in the cell.

\section{Cancer as a mechanism of increasing the channel capacity}

Several lines of investigation suggest an increase in the source capacity in cancer cells [11,12]. Onset of cancer in a tissue is associated with genetic mutations either in a single cell or multiple cells [13] followed by activation of new genes (e.g. oncogenes) and/or increased expression of already active genes $[11,12,14,15]$. In fact, each cancer cell may contain thousands of mutations at any give point in time [16]. Furthermore, aneuploidy, a state in which a cell usually harbours multiple copies of same chromosomes, is a common feature of virtually all types of cancers $[17,18]$. Aneuploidy can increase the information overload by introducing multiple copies of active genes of a cell.

When mutating agents and malfunctions such as aneuploidy alter the source capacity such that the existing $\boldsymbol{N}$-pool/AA-pool is not appropriately tuned to express the altered demand and/or set of the active genes with certain minimum error rate [6,7], the information homeostasis is disturbed. Indeed, increase in transcription and translation errors can cause multiple types of cancers $[19,20]$.

To correct for the altered information homeostasis, cells can either update the composition of the $\boldsymbol{N}$-pool and $\boldsymbol{A A - p o o l}$ or can divide to introduce parallel channels in order to distribute the information overload. In a mature cell population, cell division and introduction of a parallel information channel is a simpler strategy to restore the information homeostasis. It is important to have asymmetric cell divisions in a state of altered information homeostasis to redistribute the increased information load. It is interesting to note that the cellular machinery that promotes asymmetric cell division also plays a role in tumour suppression [8,21]. If the mutating agents activate a class of genes that cannot be optimally expressed by a certain type of specialized cells, we hypothesize that the cells would enter in a state of continuous division in an attempt to restore the information homeostasis, resulting in a cancerous tissue.

It is also possible that besides the genetic mutations and increase in source capacity, the composition of the $\boldsymbol{N - p o o l / A A - p o o l}$ is altered in cancer cells, resulting in more 'translation' errors and altered information homeostasis. That is, carcinogens can also induce cancer phenotype by reducing the channel capacity. Thus, according to our model, altered composition of the $\mathbf{N}$-pool/AA-pool can also initiate uncontrolled cell division. Therefore, a comparison of the $\mathbf{N - p o o l / A A - p o o l ~ c o m p o s i t i o n ~ o f ~ a ~ c a n c e r ~ c e l l ~ a n d ~ a ~ h e a l t h y ~}$ cell from the same tissue could provide a good test of our model.

In summary, our model suggests that in cancer cells there should be more translation and/or transcription errors which can be observed in the form of increased concentration of tRNAs and mRNAs. Our model also suggests that killing the malignant cells is not an optimal therapeutic measure against cancer, because the malfunction is not cell specific, rather it is a malfunction of a population of cells. An optimal strategy, according to the model, to control cancer would be to match the composition of nucleotide and amino-acid pools so that genetic information can be expressed with acceptable error rate.

\section{Indications of onset of cancer}

Based on our hypothesis we suggest that following early indications that can be used to predict onset of cancer.

- Sudden increase in the set of active genes in a cell population: Because this will directly change the source capacity.

- Altered composition of AA-pool: Because this will change the channel capacity.

- Malfunction in the error correction machinery in the cells: Errors in gene expression is an indicative of altered information homeostasis. Therefore, even when there is no change in source and channel capacity, increase in gene expression error would indicate altered information homeostasis and initiate cell division.

- Breakdown in mechanisms that drive asymmetric cell division: Once the information homeostasis is disturbed, cell division can restore it only if the information load can be redistributed over new cells. Asymmetric cell division is a prominent mechanism that can redistributed the information load. So, break down of asymmetric cell division would prevent restoring of the information homeostasis.

\section{Cell degeneration as a mechanism of decreasing the channel capacity}

Our model can also provide a potential explanation for cell degeneration in a tissue. Cell death effectively takes out an information channel from a tissue and from the perspective of expression of genetic information, reduces the channel capacity of a cell population. We hypothesize that in a cell population undergoing uncontrolled degeneration information homeostasis is disturbed due to decreased source capacity. Thus, we hypothesize that in such a degenerating cell population a large fraction of the active genes may have gone silent. This could also occur due to the change in the environmental cues such that only few cells are sufficient to express active genes of the cell population in required demand. Maintaining cells requires energy and it is more efficient to kill a cell if it is not required to express any information or if the energy cost of information has exceeded a certain limit. A test of this hypothesis could be that in a cell population undergoing degeneration there should less active genes or other mechanisms which suppress the gene expression, should be hyperactive. Indeed, in Alzheimer's disease there is general reduction in gene expression in CA1 region of the brain [22]. Similarly, in amyotrophic lateral sclerosis more genes are down-regulated than upregulated [23]. Furthermore, down-regulation of a key protein 'parkin' precedes death of affected neurons in cerebral ischemia, and up-regulation of this gene can prevent the neurons from dying [24]. In general there is a reorganization of gene expression in the cells of a degenerating tissue (e.g. in Huntington's disease [25]). However, a simple estimate of up and down-regulated genes is not sufficient to conclude decrease in source capacity in degenerating tissue. More systematic analysis of the amino-acid requirements of up and down-regulated genes would be necessary to validate or refute our hypothesis.

\section{Evaluation of the model}

Although, our phenomenological model gives a description of the cell function beyond the exact details of the biochemical 
interactions, yet, we can make testable prediction to validate or refute our hypothesis.

1. One of the explicit tests of the model would be to check if increasing the number of active genes in a cell population makes it divide at a higher rate than without the newly activated genes. Alternatively, it would be important to quantify the effect of altering the composition of the pool of amino-acids and nucleotides on the rate of cell division in a cell population.

2. Experimental observation of tissue specific $\boldsymbol{N}$-pool/AA-pool composition would support our model. Cells in different tissues express different sets of the genes depending on the environmental cues and active genes [4]. In order to maintain information homoeostasis each cell should have an optimal composition of the $\mathbf{N - p o o l / A A - p o o l , ~ d i f f e r e n t ~ f o r ~ c e l l s ~ f r o m ~}$ other tissues.

3. Our model predicts that information homoeostasis is altered in the cancer cells such that the source capacity is larger than the channel capacity. This means that in cancer cells there should be a higher proportion of mRNAs which could not find a matching amino-acid. Previous work already provides partial support for this prediction [26]. A high count of defective ribosomal product [27] in cancer cells compared to healthy cells would be consistent with our model.

4. Using the model, we also predict a significant change in the composition of the $\boldsymbol{N}$-pool/AA-pool in cancer and healthy cells from the same tissue. It is not possible to comment on how specifically the $\boldsymbol{A} \boldsymbol{A}$-pool composition will change and this can only be determined by careful experiments. Recent observation that nucleotide deficiency can cause cancer provides experimental support for our hypothesis [28].

5. In benign tumours, according to our model, initially there should be an imbalance of information homeostasis which gets balanced by subsequent division. Thus, the existence of benign tumours is an evidence in favour of our model.

6. In a cell population undergoing uncontrolled degeneration, we predict that there should be an increase in the activity of gene suppressing agents, because according to our model in degenerating tissue source capacity decreases significantly compared to the channel capacity.

\section{Discussion}

Based on a striking similarity between a typical digital communications system and flow of genetic information from DNA to proteins, here we propose an information theory based model of transmission of genetic information (fig. 1). We introduce the term 'information homeostasis' which ensures that genetic information is expressed with minimal error rate and refers to a state of cell in which the composition of $\boldsymbol{N}$-pool and $\boldsymbol{A A}$-pool is optimally matched to the requirements of the active genes in a cell ( $\boldsymbol{S} \leqslant \boldsymbol{C}_{\text {eff }}$ ). Using this model, we argue that together with 'energy homeostasis', 'information homeostasis' is a fundamental working principle of the biological cells.

The model suggests that cell division is a strategy to increase the channel capacity by introducing additional information channels i.e. each new cell is a potential new channel for expression of genetic information. Similarly, cell death is a way to reduce the channel capacity. Thus, our model suggests that cell division and cell death are mechanisms of maintaining the information homoeostasis in a given cell population. The model also provides novel insights about the uncontrolled cell division and cell death and makes testable predictions that can validate (or refute) the model. Finally, our model puts cell division/death in the broader context of error-free expression of genetic information and offers an answer to the question as to why cells divide in an otherwise stable multicellular environment such as a tissue. We note that this hypothesis does not explain why a cell in a normal tissue, undergoing normal cell cycle divides. Our hypothesis is an attempt to develop a conceptual framework to understand increased cell division or cell death in a cell population.

Beyond cancer and degenerative diseases the model can be used to understand several related phenomena such organ size. When a part of a mature liver is surgically removed, the organ regains its full size. Often the "total-mass checkpoint" hypothesis is invoked to explain this phenomenon [29]. We think that this phenomenon reveals something about the information homeostasis that a liver cell population is supposed to maintain. In a state when the gene expression determined by the environmental cues is largely error-free the cell division and cell death are balanced to maintain the size of the organ. However, when a part is removed, the overall capacity of the cell population is reduced and cell divisions start to create additional channels to express the required genetic information, and such cell division stops when information homeostasis is regained. Thus, our hypothesis may also provide insights into the process of organ size maintenance.

Our phenomenological model can also provide a framework to understand similarities between an embryo and a cancer cell. Intriguingly, there are multiple similarities between an embryo and a cancerous tissue such as damaged DNA, common trigger for deprogramming, and the cessation of informational signalling [30]. Here, we suggest that there is a more fundamental similarity between a cancerous tissue and the embryo in terms of genetic information overload. In the framework of our model, an embryo is also in a state of altered information homeostasis. The embryo has a lot of genomic information that cannot be expressed by a single cell. Therefore, subsequent divisions (which are generally asymmetric) of the embryo and the emergent differentiation of daughter cells can be viewed as a process of maintaining the information homeostasis by distribution of the genetic information expression over new channels. Here we do not mean to suggest that embryos and cancer cells are alike, but the similarities between the two are conspicuous and go beyond sharing of specific biochemical signalling. Activation of embryonic genes in the cancerous cells [30] suggest that these genes have some similarity with the oncogenes in terms of their requirements of nucleotides and amino-acids, which drives the cells into an information overload. Thus, we also suggest that some oncogenes would be active in the embryonic cells. A systematic analysis of the statistical properties of genes would be needed to identify such similarities among embryonic genes and oncogenes.

In summary, here we have proposed the hypothesis of 'information homeostasis' which we think is one of the general principles governing cell division in a multi-cellular organism. This hypothesis makes several testable predictions and provides a conceptual understanding of different malfunctions related to altered cell division and apoptosis.

\section{Conflict of interest}

None declared.

\section{Acknowledgement}

We thank Dr. M. Asif Arif, Dr. Smitha Vishnu, Dr. Anushree Sanyal and Malikmohamed Yousuf for discussions and comments on the manuscript.

\section{Reference}

[1] Morgan DO. The cell cycle: principles of control. London, UK: New Science Press Ltd.; 2007. 
[2] Melino G, Vaux D. Cell Death. Chichester, UK: John Wiley and Sons; 2010.

[3] Shannon CE. A mathematical theory of communication. Bell Syst. Tech. J. 1948:27379-423.

[4] Schmid M, Davison TS, Henz SR, Pape UJ, Demar M, Vingron M, et al. A gene expression map of Arabidopsis thaliana development. Nat Genet 2005;37(5):501-6

[5] Rosenberger RF, Foskett G. An estimate of the frequency of in vivo transcriptional errors at a nonsense codon in Escherichia coli. Mol Gen Genet $1981 ; 183(3): 561-3$.

[6] Ninio J. Connections between translation, transcription and replication errorrates. Biochimie 1991;73(12):1517-23.

[7] Drummond DA, Wilke CO. The evolutionary consequences of erroneous protein synthesis. Nat. Rev. Genet. 2009;10(10):715-24.

[8] Morrison SJ, Kimble J. Asymmetric and symmetric stem-cell divisions in development and cancer. Nature 2006;441(7097):1068-74.

[9] Hawkins N, Garriga G. Asymmetric cell division: from A to Z. Genes Dev 1998:12(23):3625-38.

[10] Hawkins ED, Russell SM. Upsides and downsides to polarity and asymmetric cell division in leukemia. Oncogene 2008;27(55):7003-17.

[11] Zhang L, Zhou W, Velculescu VE, Kern SE, Hruban RH, Hamilton SR, et al. Gene expression profiles in normal and cancer cells. Science 1997;276(5316):1268-72.

[12] Chapman MA, Lawrence MS, Keats JJ, Cibulskis K, Sougnez C, Schinzel AC, et al. Initial genome sequencing and analysis of multiple myeloma. Nature 2011;471(7339):467-72.

[13] Knudson AG. Two genetic hits (more or less) to cancer. Nat Rev Cancer 2001:1157-62.

[14] Cao D, Hustinx SR, Sui G, Bala P, Sato N, Martin S, et al. Identification of nove highly expressed genes in pancreatic ductal adenocarcinomas through a bioinformatics analysis of expressed sequence tags. Cancer Biol Ther 2004;3(11):1081.

[15] Han H, Bearss DJ, Browne LW, Calaluce R, Nagle RB, Hoff DDV. Identification of differentially expressed genes in pancreatic cancer cells using cDNA microarray. Cancer Res 2002;62(10):2890-6.

[16] Loeb LA. A Mutator Phenotype in Cancer. Cancer Res 2001:613230-9.
[17] Duesberg P, Rasnick D. Aneuploidy, the somatic mutation that makes cancer a species of its own. Cell Motil Cytoskeleton 2000:47(2):81-107.

[18] Rajagopalan H, Lengauer C. Aneuploidy and cancer. Nature 2004:432338-41.

[19] Chen L, Madura K. Increased proteasome activity, ubiquitin-conjugating enzymes, and eEF1A translation factor detected in breast cancer tissue. Cancer Res 2005;65(13):5599-606.

[20] Hoeijmakers JH. Genome maintenance mechanisms for preventing cancer. Nature 2001;411(6835):366-74.

[21] Clevers H. Stem cells, asymmetric division and cancer. Nat Genet 2005;37(10):1027-8.

[22] Colangelo V, Schurr J, Ball MJ, Pelaez RP, Bazan NG, Lukiw WJ. Gene expression profiling of 12633 genes in Alzheimer hippocampal CA1: transcription and neurotrophic factor down-regulation and up-regulation of apoptotic and proinflammatory signaling. J Neurosci Res 2002;70(3):462-73.

[23] Jiang Y-M, Yamamoto M, Kobayashi Y, Yoshihara T, Liang Y, Terao S, et al. Gene expression profile of spinal motor neurons in sporadic amyotrophic latera sclerosis. Ann Neurol 2005;57(2):236-51.

[24] Mengesdorf T, Jensen PH, Mies G, Aufenberg C, Paschen W. Down-regulation of parkin protein in transient focal cerebral ischemia: A link between stroke and degenerative disease? Proc Natl Acad Sci USA 2002;99(23):15042-7.

[25] Hodges A, Strand AD, Aragaki AK, Kuhn A, Sengstag T, Hughes G, et al. Regional and cellular gene expression changes in human Huntington's disease brain. Hum Mol Genet 2006;15(6):965-77.

[26] Loeb LA, Springgate CF, Battula N. Errors in DNA replication as a basis of malignant changes. Cancer Res 1974;34(9):2311-21.

[27] Schubert U, Antón LC, Gibbs J, Norbury CC, Yewdell JW, Bennink JR. Rapid degradation of a large fraction of newly synthesized proteins by proteasomes. Nature 2000;404(6779):770-4.

[28] Bester AC, Roniger M, Oren YS, Im MM, Sarni D, Chaoat M, et al. Nucleotide deficiency promotes genomic instability in early stages of cancer development. Cell 2011;145:435-46

[29] Potter CJ, Xu T. Mechanisms of size control. Curr Opin Genet Dev $2001 ; 11(3): 279-86$.

[30] Monk M, Holding C. Human embryonic genes re-expressed in cancer cells. Oncogene 2001;20(56):8085-91. 